\section{Our Experiences with Bi-Planar Mastopexy-Augmentation}

\section{Recep Anlatıcı*}

Department of Aesthetic, Plastic and Reconstructive Surgery, Sanko University, Gaziantep, Turkey

\begin{abstract}
Aims: By observing some restrictions with the widely performed mastopexy-augmentation operation solely involving the subglandular plane, we modified the method into a two-plane intervention in a number of selected cases. In this retrospective clinical study, we aimed to share our experiences with single stage bi-planar mastopexy-augmentation.

Methods: We performed a vertical mammoplasty and dissected both the subglandular plane, to fix the glandular tissue to a higher pectoral fascial level, and the submuscular plane to insert the implant. Additionally, we utilized either the superior-median dermofat or the dermoglandular flap to cover the implants completely or incompletely, aiming for a more stabilized vertical closure. The results were assessed retrospectively and statistically.

Results: Thirty-six cases (72 breasts) were included in the study. The average age was 42 years and the average follow-up period was 13 months. The mean sizes of the implants were $211.81 \pm 67.48 \mathrm{cc}$ for the right breasts and $213.19 \pm 66.41 \mathrm{cc}$ for the left breasts. Twenty-eight cases $(77.78 \%)$ were classified as primary and the remaining eight $(22.22 \%)$ as secondary. Revision operations were demanded in five $(13.89 \%)$ cases, three $(10.71 \%)$ in the primary and two $(25 \%)$ in the secondary group. Postoperative complications were observed in $50 \%$ of the study population and also $50 \%$ of each group. However, whereas all complications were identified as major in the secondary group, only three cases $(10.71 \%)$ of major complications were reported in the primary group. There was a significant statistical relationship between the grade of ptosis and minor complications (for which the grade 3 ptosis group was responsible). Problems due to previous breast operations of the secondary group were significantly correlated with the major problems in our study population. However,
\end{abstract}

*Corresponding author: Recep Anlatıcı, Department of Aesthetic, Plastic and Reconstructive Surgery, Sanko University, Gaziantep, Turkey, Tel: +90 05326760599, E-mail: recepanlatici@hotmail.com

Citation: Anlatıcı R (2019) Our Experiences with Bi-Planar Mastopexy-Augmentation, J Surg Curr Trend Innov 3: 019.

Received: August 14, 2019; Accepted: August 29, 2019; Published: September 05, 2019

Copyright: (c) 2019 Anlatıcı R. This is an open-access article distributed under the terms of the Creative Commons Attribution License, which permits unrestricted use, distribution, and reproduction in any medium, provided the original author and source are credited. the grade of ptosis, implant volume, and previous mastopexy-augmentation operation were not related with the revision operations and postoperative complications.

Conclusion: We believe that, in select cases, it is feasible to perform bi-planar mastopexy-augmentation to overcome the limitations of the widely performed one-plane method. However, potential postoperative complications should be taken into consideration and more caution in secondary cases is needed due to higher complication rates.

Keywords: Bi-planar mastopexy-augmentation; Complications of mastopexy-augmentation; Single stage mastopexy-augmentation

\section{Introduction}

The safety of breast augmentation and mastopexy performed as a single procedure has often been debated [1-5]. As stated by Khavanin et al, [3] the literature regarding this procedure is relatively sparse; however, several cases using vertical or inverted T mastopexy with insertion of either a single or dual plane implant have been reported recently $[4,6-8]$. We are among those who perform the mastopexy-augmentation operation in a single stage. Faced with some restrictions with the widely performed sole submammarial plane procedure in some cases, we modified the method by creating two pockets: The first as a subglandular plane to obtain stable and effective lifting of the breast tissue (with Lejour fixing suture); and the latter as a submuscular plane to insert the implant (Figure1). In this retrospective clinical study, we aimed to share our experiences with single stage bi-planar mastopexy-augmentation.

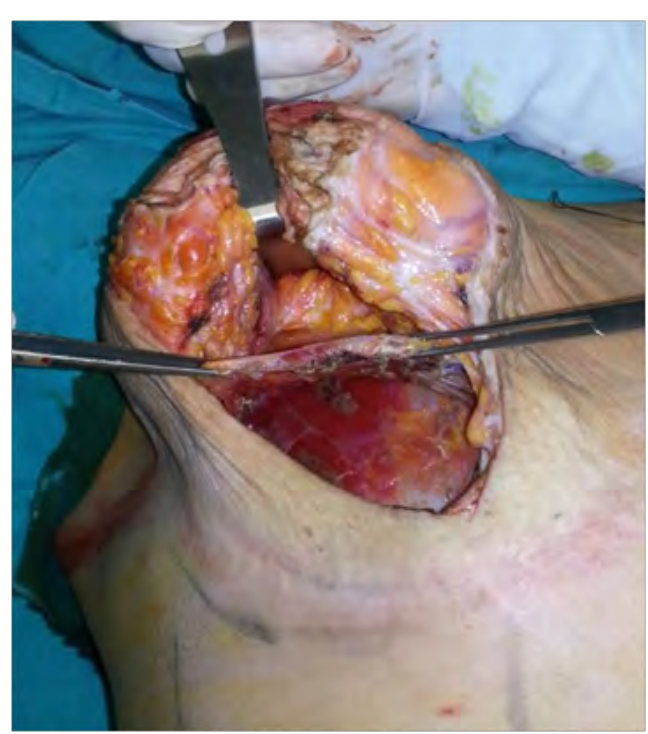

Figure 1: Submuscular and subglandular pockets during superior pedicle vertical mastopexy-augmentation. The pectoralis muscle is grasped and elevated, and the glandular tissue is retracted. 


\section{Materials and Methods}

Within each case, several parameters were investigated, these included: Patient age, preoperative diagnosis with grade of ptosis, history of previous breast augmentation operations (to classify the cases as primary or secondary), follow-up period, revisions, implant types and volumes, and postoperative complications (recurrent ptosis, capsule contracture, wound opening, implant exposure, hematoma, asymmetries, scar etc.). Licensed fourth generation, smooth or textured, high or moderate profile, cohesive gel implants were used in all cases. All patients were fully informed about the operation, including the possible complications and results, prior to giving signed consent. The sizes of the implants were based on both the patients' preference and physical examination (which assessed the laxity of the breast, degree of ptosis, preoperative volume of the breast, and the general physical properties of the patients) as well as an intraoperative evaluation using implant sizers.

Double pocket mastopexy-augmentation was employed in cases where the classical single plane method was not possible; the restriction of the subglandular space following Lejour's fixing suture $[9,10]$ and existence of severe capsular contraction in secondary cases were the two main factors. We used a vertical mammaplasty technique and fixed the breast tissue at the areola-nipple level to the uppermost part of the pectoral fascia as described by Lejour; $[9,10]$ the rationale behind the use of this technique was to improve the stability of the results and gain increased fullness in the upper portion of the breasts The implants were placed in the subpectoral pockets after the medial-inferior origins of the pectoralis muscles were detached. Evaluation of the tightness of the vertical suture line and the decision on the necessity of additional skin removal was made by testing different volumes of implant sizers and temporary staplers. Limited elevation of the skin edges on the lateral pillars was carried out if required in order to reduce the tension on the suture lines. Placing the implant in the desired position enabled the superomedian dermoglandular or dermofat flap to cover the implant completely or incompletely (Figure 2 ); this reduced the risk of implant injury during wound closure of the wound or, with regards to postoperative wound healing, the risk of implant exposure.

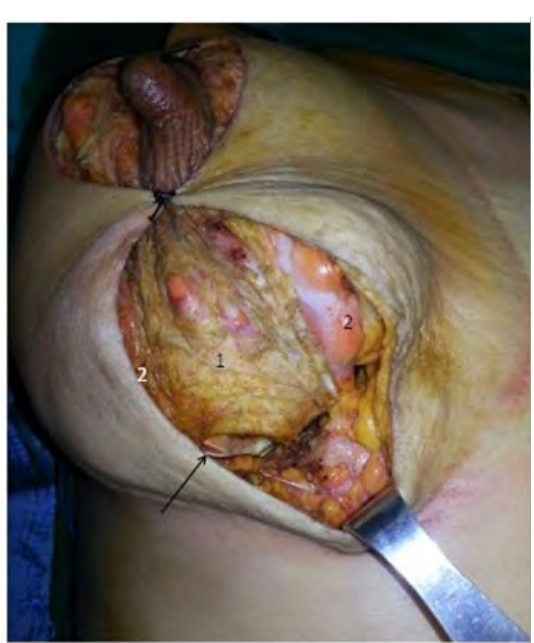

Figure 2: The superomedian flap and the lateral pillars are indicated as 1 and 2, respectively. The implant (arrow) is covered with the superomedian flap.
This method was also used for secondary cases in order to treat complications such as recurrent ptosis with or without capsular contracture. In all cases we detected older generation implants (ruptured or not) that were placed in the subglandular plane; these implants were removed and the pockets were copiously irrigated to remove the free silicone, if present. A limited or partial capsulectomy was carried out, unless there was evidence of calcification or any other severe complication associated with the subglandular capsule. We frequently use the capsule as a strong and stable tissue to lift and fix the breast tissue to the pectoralis fascia, and also for steadier wound closure. Problems generated by subglandular capsular contracture were simply solved by changing the plane for the new implants to the submuscular pocket.

\section{Statistical Analysis}

The mean and standard deviation values are given for continuous variables indicated by measurement; frequency and percentage values are given for qualitative variables. In group comparisons, in the case of parametric test conditions for continuous variables indicated by measurement, an independent sample t-test was used to analyze the difference between the two means. A Chi-squared test and Fisher's exact test were used for group comparisons of qualitative variables. The significance of $p$ value was set at 0,05 .

\section{Results}

We performed biplanar mastopexy-augmentation in 36 cases ( 72 breasts) (Figures 3-6). The average age of the patients was 42 years old and the average follow-up period was 9 months. Additional procedures such as liposuction, abdominoplasty, or extremity lifting were carried out in 13 cases $(36.11 \%)$. Twenty-eight cases $(77.78 \%)$ were classified as primary and the remaining $8(22.22 \%)$ as secondary (Tables 1 and 2). Six out of the eight secondary cases (75\%) were admitted to our clinic with severe capsular contracture and/or malposition of the implant. Eight cases (22.22\%) were diagnosed with Regnault level 1 ptosis, eleven (30.56\%) with level 2, eight (22.22\%) with level 3, and nine (25\%) with pseudoptosis. Implants between 100 and $400 \mathrm{cc}$ were used for these cases, with mean values of $211.81 \pm 67.48 \mathrm{cc}$ for the right breasts and $213.19 \pm 66.41 \mathrm{cc}$ for the left breasts. The implant volumes of the secondary cases were significantly greater than those of the primary cases (Table 3). Revision operations were demanded in five $(13.89 \%)$ cases (Table 4$)$; this included three cases $(10.71 \%)$ in the primary group and two cases $(25 \%)$ in the secondary group. Revisions were carried out in three cases $(8.33 \%)$ for asymmetry, in one case $(2.78 \%)$ for capsule contracture and asymmetry, and in one case $(2.78 \%)$ for the presence of a widened scar.

Postoperative complications were observed in half of the study population (Table 4). Implant exposure, a major complication, was observed in two $(5.56 \%)$ cases. Other major complications were seen in one $(2.78 \%)$ case at a time and included asymmetry, severe capsular contracture with resultant asymmetry, hematoma, and wound opening that was managed by surgery, and wound opening with secondary healing. Minor complications were also noted and included mild asymmetry $(n=4,11.11 \%)$, mild sagging $(n=5,13.89 \%)$, widened scar $(n=1,2.78 \%)$, and wound healing issues with secondary epithelization $(\mathrm{n}=1,2.78 \%)$. Postoperative complications were observed in $50 \%$ of the 28 primary cases; of these, eleven $(30.56 \%)$ were defined as minor complications and three $(10.71 \%)$ as major complications. Postoperative complications were also observed in half of the 
secondary group; however, all of these were defined as major complications. In the cases with implant exposure, implants were removed and new implants were reinserted after a delayed period. In the cases with grade 3 capsule contraction, implants were replaced in parallel to performing a capsulectomy.

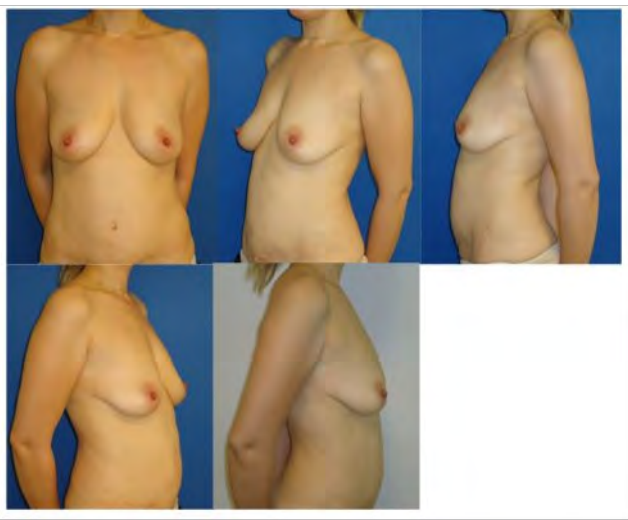

Figure 3: Preoperative images of case 28

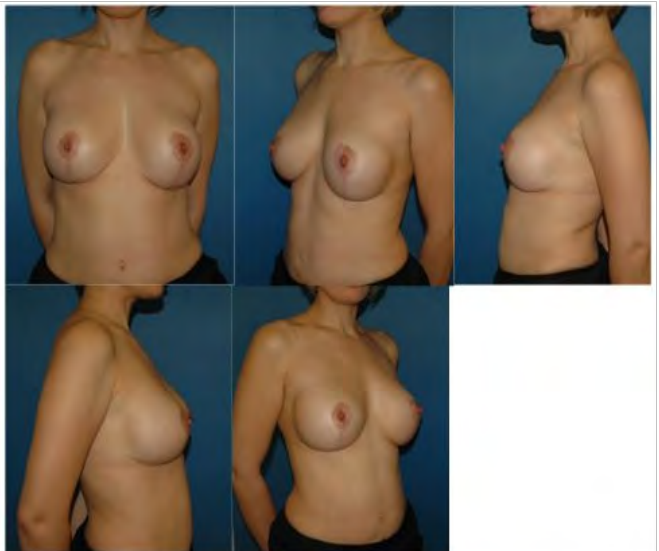

Figure 4: Postoperative images of case 28 after 12 months (250cc smooth high-profile implants).

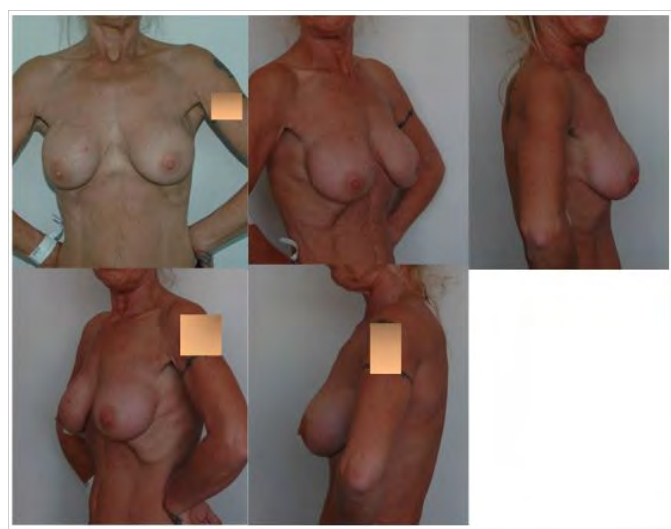

Figure 5: Preoperative images of case 29, who was admitted with deformities due to capsule contraction after an augmentation procedure. Implants were removed with their surrounding capsules (small image, bottom right).

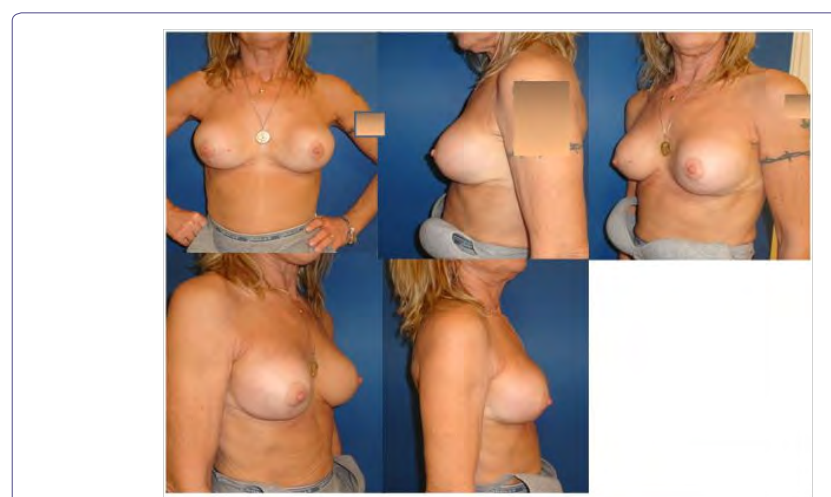

Figure 6: Postoperative images of case 29 after 22 months (smooth high profile $2 \times 250 \mathrm{cc}$ new implants).

\begin{tabular}{|c|c|c|c|c|}
\hline Case \# & Diagnosis and Grade of ptosis & $\begin{array}{l}\text { Primary (P) or } \\
\text { Secondary (S) }\end{array}$ & $\begin{array}{c}\text { Implant ce } \\
\text { (right) }\end{array}$ & $\begin{array}{l}\text { Implant } \\
\text { ce (left) }\end{array}$ \\
\hline 1 & 3 & $P$ & 100 & 100 \\
\hline 2 & 1 & $\mathrm{P}$ & 125 & 125 \\
\hline 3 & 1 & $\mathrm{P}$ & 125 & 125 \\
\hline 4 & 2 & $\mathrm{P}$ & 125 & 125 \\
\hline 5 & Pseudoptosis & $\mathrm{P}$ & 125 & 125 \\
\hline 6 & 1 & $\mathrm{P}$ & 150 & 175 \\
\hline 7 & 1 & $\mathrm{P}$ & 150 & 175 \\
\hline 8 & 1 & $\mathrm{~s}$ & 150 & 150 \\
\hline 9 & 3 & $\mathrm{P}$ & 150 & 150 \\
\hline 10 & pseudoptosis and gr 3 contracture & $\mathrm{S}$ & 150 & 150 \\
\hline 11 & 2 & $P$ & 175 & 175 \\
\hline 12 & 3 & $\mathrm{P}$ & 175 & 200 \\
\hline 13 & 3 & $\mathrm{P}$ & 175 & 150 \\
\hline 14 & 3 & $P$ & 175 & 175 \\
\hline 15 & pseudoptosis & $\mathrm{P}$ & 175 & 175 \\
\hline 16 & 2 & $P$ & 200 & 200 \\
\hline 17 & 2 & $\mathrm{P}$ & 200 & 200 \\
\hline 18 & 3 & $P$ & 200 & 200 \\
\hline 19 & 1 & $\mathrm{P}$ & 225 & 225 \\
\hline 20 & 2 & $\mathrm{P}$ & 225 & 250 \\
\hline 21 & 2 & $P$ & 225 & 225 \\
\hline 22 & Pseudoptosis & $\mathrm{P}$ & 225 & 225 \\
\hline 23 & 2 & $P$ & 250 & 250 \\
\hline 24 & 2 & $P$ & 250 & 250 \\
\hline 25 & 2 & $\mathrm{P}$ & 250 & 250 \\
\hline 26 & 2 & $P$ & 250 & 250 \\
\hline 27 & 3 & $\mathrm{P}$ & 250 & 250 \\
\hline 28 & 3 & $\mathrm{P}$ & 250 & 250 \\
\hline 29 & capsule contracture, malposition & $\mathrm{s}$ & 250 & 250 \\
\hline 30 & capsule contracture & $\mathrm{S}$ & 250 & 250 \\
\hline 31 & 2 & $\mathrm{P}$ & 275 & 250 \\
\hline 32 & pseudoptosis and gr 3 contracture & $\mathrm{s}$ & 275 & 275 \\
\hline 33 & 1 , malposition & $\mathrm{s}$ & 300 & 300 \\
\hline 34 & 1 & $\mathrm{P}$ & 300 & 300 \\
\hline 35 & pseudoptosis and gr 3 contracture & $\mathrm{S}$ & 350 & 350 \\
\hline 36 & Pseudoptosis & $\mathrm{S}$ & 400 & 400 \\
\hline
\end{tabular}

Table 1: Diagnosis, grade of ptosis, groups of cases, and implant volumes. 


\begin{tabular}{|c|c|c|c|c|c|}
\hline \multicolumn{6}{|c|}{ Descriptive Statistics } \\
\hline & $\mathrm{N}$ & Minimum & Maximum & Mean & Std. Deviation \\
\hline Implant cc R & 36 & 100 & 400 & \multirow[b]{2}{*}{213,19} & 67,476 \\
\hline Implant cc L & 36 & 100 & 400 & & 66,409 \\
\hline Valid N (listwise) & \multicolumn{2}{|l|}{36} & & & \\
\hline \multicolumn{3}{|c|}{ Primary (P) and Secondary (S) Groups } & \multicolumn{2}{|c|}{ Implant ce R } & Implant ce L \\
\hline \multirow{3}{*}{ Primary group } & & $\mathrm{N}$ & \multicolumn{2}{|c|}{28} & 28 \\
\hline & \multicolumn{2}{|r|}{ Mean } & \multicolumn{2}{|c|}{196,43} & 198,21 \\
\hline & \multicolumn{2}{|c|}{ Std. Deviation } & \multicolumn{2}{|c|}{53,017} & 51,787 \\
\hline \multirow{3}{*}{ Secondary group } & & $\mathrm{N}$ & \multicolumn{2}{|c|}{8} & 8 \\
\hline & \multicolumn{2}{|r|}{ Mean } & \multicolumn{2}{|c|}{265,63} & 265,63 \\
\hline & \multicolumn{2}{|c|}{ Std. Deviation } & \multicolumn{2}{|c|}{87,564} & 87,564 \\
\hline \multirow{3}{*}{ Total } & & $\mathrm{N}$ & \multicolumn{2}{|c|}{36} & 36 \\
\hline & \multicolumn{2}{|r|}{ Mean } & \multicolumn{2}{|c|}{211,81} & 213,19 \\
\hline & \multicolumn{2}{|c|}{ Std. Deviation } & \multicolumn{2}{|c|}{67,476} & 66,409 \\
\hline
\end{tabular}

Table 2: Descriptive statistics regarding breast implant sizes (R: right, L: left).

\begin{tabular}{|c|c|c|c|c|l|}
\hline \multicolumn{7}{|c|}{ Group Statistics } \\
\hline \multirow{3}{*}{} & Primary (P) or Secondary (S) & N & Mean & $\begin{array}{c}\text { Std. Devi- } \\
\text { ation }\end{array}$ & $\begin{array}{l}\text { Std. Error } \\
\text { of Mean }\end{array}$ \\
\hline & Primary & 28 & 196,43 & 53,017 & 10,019 \\
\cline { 2 - 6 } & Secondary & 8 & 265,63 & 87,564 & 30,958 \\
\hline \multirow{2}{*}{$\begin{array}{c}\text { Implant } \\
\text { cc L }\end{array}$} & Primary & 28 & 198,21 & 51,787 & 9,787 \\
\cline { 2 - 6 } & Secondary & 8 & 265,63 & 87,564 & 30,958 \\
\hline
\end{tabular}

Table 3: Implant volumes are significantly increased in the secondary group compared to the primary group $(\mathrm{p}<0.05)$.

\begin{tabular}{|c|c|c|c|}
\hline $\begin{array}{c}\text { Case } \\
\#\end{array}$ & $\begin{array}{c}\text { Revision performed } \\
\text { for }\end{array}$ & $\begin{array}{c}\text { Major postoperative com- } \\
\text { plications }\end{array}$ & $\begin{array}{c}\text { Minor postoperative } \\
\text { complications }\end{array}$ \\
\hline 1 & Widened scar & & Widened scar \\
\hline 5 & Asymmetry & & Mild Asymmetry \\
\hline 7 & & & Mild Asymmetry \\
\hline 9 & & $\begin{array}{c}\text { Wound opening managed by } \\
\text { operation + asymmetry }\end{array}$ & Mild Asymmetry \\
\hline 10 & & & Mild sagging \\
\hline 11 & & & Mild sagging \\
\hline 13 & & Implant exposure & Mild sagging \\
\hline 14 & & Asymmetry & \\
\hline 16 & & Hematoma & \\
\hline 17 & Asymmetry & & Mild sagging \\
\hline 23 & & & Mild Asymmetry \\
\hline 25 & & & Wound problem with \\
\hline 26 & & Implant exposure & Mild sagging \\
\hline 27 & & & \\
\hline 28 & & asymmetry & \\
\hline 32 & & $\begin{array}{c}\text { Wound problem with second- } \\
\text { ary healing + asymmetry }\end{array}$ & \\
\hline 33 & Capsule contracture gr & \begin{tabular}{c} 
Capsule contracture grade + \\
\hline 35
\end{tabular} & Asymmetry \\
\hline
\end{tabular}

Table 4: Revisions performed and complications observed in all cases.
We investigated whether the number of revision operations and postoperative complications (minor, major, or total) were significantly correlated with the ptosis level, implant volume, and previous mastopexy-augmentation operation (Tables 5-16). Results demonstrated a significant relationship between the grade of ptosis and the minor complications (for which the grade 3 ptosis group was responsible). Major complications were seen significantly more frequent in the secondary group. All other correlations were insignificant.

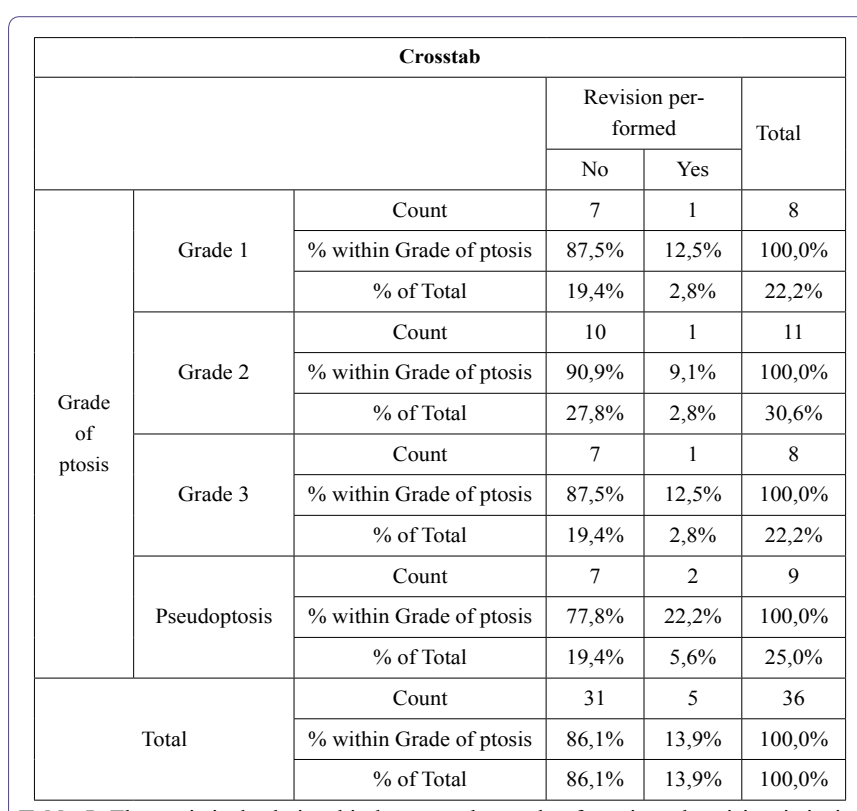

Table 5: The statistical relationship between the grade of ptosis and revisionsis insignificant $(\mathrm{p}>0,05)$.

\begin{tabular}{|c|c|c|c|c|c|}
\hline \multicolumn{6}{|c|}{ Crosstab } \\
\hline & & & \multicolumn{2}{|c|}{$\begin{array}{l}\text { Major postopera- } \\
\text { tive complications }\end{array}$} & \multirow[t]{2}{*}{ Total } \\
\hline & & & No & Yes & \\
\hline \multirow{12}{*}{$\begin{array}{l}\text { Grade } \\
\text { of } \\
\text { ptosis }\end{array}$} & \multirow{3}{*}{ Grade 1} & Count & 7 & 1 & 8 \\
\hline & & $\%$ within Grade of ptosis & $87,5 \%$ & $12,5 \%$ & $100,0 \%$ \\
\hline & & $\%$ of Total & $19,4 \%$ & $2,8 \%$ & $22,2 \%$ \\
\hline & \multirow{3}{*}{ Grade 2} & Count & 8 & 3 & 11 \\
\hline & & $\%$ within Grade of ptosis & $72,7 \%$ & $27,3 \%$ & $100,0 \%$ \\
\hline & & $\%$ of Total & $22,2 \%$ & $8,3 \%$ & $30,6 \%$ \\
\hline & \multirow{3}{*}{ Grade 3} & Count & 8 & 0 & 8 \\
\hline & & $\%$ within Grade of ptosis & $100,0 \%$ & $0,0 \%$ & $100,0 \%$ \\
\hline & & $\%$ of Total & $22,2 \%$ & $0,0 \%$ & $22,2 \%$ \\
\hline & \multirow{3}{*}{ Pseudoptosis } & Count & 6 & 3 & 9 \\
\hline & & $\%$ within Grade of ptosis & $66,7 \%$ & $33,3 \%$ & $100,0 \%$ \\
\hline & & $\%$ of Total & $16,7 \%$ & $8,3 \%$ & $25,0 \%$ \\
\hline \multirow{3}{*}{\multicolumn{2}{|c|}{ Total }} & Count & 29 & 7 & 36 \\
\hline & & $\%$ within Grade of ptosis & $80,6 \%$ & $19,4 \%$ & $100,0 \%$ \\
\hline & & $\%$ of Total & $80,6 \%$ & $19,4 \%$ & $100,0 \%$ \\
\hline
\end{tabular}

Table 6: Statistical relationship between grade of ptosis and major postoperativecomplications is insignificant $(\mathrm{p}>0,05)$. 
Citation: Anlatıcı R (2019) Our Experiences with Bi-Planar Mastopexy-Augmentation, J Surg Curr Trend Innov 3: 019.

- Page 5 of 8 •

\begin{tabular}{|c|c|c|c|c|c|}
\hline \multicolumn{6}{|c|}{ Crosstab } \\
\hline & & & \multicolumn{2}{|c|}{$\begin{array}{l}\text { Minor postopera- } \\
\text { tive complications }\end{array}$} & \multirow{2}{*}{ Total } \\
\hline & & & No & Yes & \\
\hline \multirow{12}{*}{$\begin{array}{l}\text { Grade } \\
\text { of } \\
\text { ptosis }\end{array}$} & \multirow{3}{*}{ Grade 1} & Count & 7 & 1 & 8 \\
\hline & & $\%$ within Grade of ptosis & $87,5 \%$ & $12,5 \%$ & $100,0 \%$ \\
\hline & & $\%$ of Total & $19,4 \%$ & $2,8 \%$ & $22,2 \%$ \\
\hline & \multirow{3}{*}{ Grade 2} & Count & 8 & 3 & 11 \\
\hline & & $\%$ within Grade of ptosis & $72,7 \%$ & $27,3 \%$ & $100,0 \%$ \\
\hline & & $\%$ of Total & $22,2 \%$ & $8,3 \%$ & $30,6 \%$ \\
\hline & \multirow{3}{*}{ Grade 3} & Count & 2 & 6 & 8 \\
\hline & & $\%$ within Grade of ptosis & $25,0 \%$ & $75,0 \%$ & $100,0 \%$ \\
\hline & & $\%$ of Total & $5,6 \%$ & $16,7 \%$ & $22,2 \%$ \\
\hline & \multirow{3}{*}{ Pseudoptosis } & Count & 8 & 1 & 9 \\
\hline & & $\%$ within Grade of ptosis & $88,9 \%$ & $11,1 \%$ & $100,0 \%$ \\
\hline & & $\%$ of Total & $22,2 \%$ & $2,8 \%$ & $25,0 \%$ \\
\hline \multirow{3}{*}{\multicolumn{2}{|c|}{ Total }} & Count & 25 & 11 & 36 \\
\hline & & $\%$ within Grade of ptosis & $69,4 \%$ & $30,6 \%$ & $100,0 \%$ \\
\hline & & $\%$ of Total & $69,4 \%$ & $30,6 \%$ & $100,0 \%$ \\
\hline
\end{tabular}

Table 7: The statistical relationship between the grade of ptosis and minor postoperative complicationsis significant $(\mathrm{p}<0,05)$ (caused bythe grade 3 group).

\begin{tabular}{|c|c|c|c|c|c|}
\hline \multicolumn{6}{|c|}{ Crosstab } \\
\hline & & & \multicolumn{2}{|c|}{$\begin{array}{l}\text { Total postopera- } \\
\text { tive complications }\end{array}$} & \multirow[t]{2}{*}{ Total } \\
\hline & & & No & Yes & \\
\hline \multirow{12}{*}{$\begin{array}{c}\text { Grade } \\
\text { of } \\
\text { ptosis }\end{array}$} & \multirow{3}{*}{ Grade 1} & Count & 6 & 2 & 8 \\
\hline & & $\%$ within Grade of ptosis & $75,0 \%$ & $25,0 \%$ & $100,0 \%$ \\
\hline & & $\%$ of Total & $16,7 \%$ & $5,6 \%$ & $22,2 \%$ \\
\hline & \multirow{3}{*}{ Grade 2} & Count & 5 & 6 & 11 \\
\hline & & $\%$ within Grade of ptosis & $45,5 \%$ & $54,5 \%$ & $100,0 \%$ \\
\hline & & $\%$ of Total & $13,9 \%$ & $16,7 \%$ & $30,6 \%$ \\
\hline & \multirow{3}{*}{ Grade 3} & Count & 2 & 6 & 8 \\
\hline & & $\%$ within Grade of ptosis & $25,0 \%$ & $75,0 \%$ & $100,0 \%$ \\
\hline & & $\%$ of Total & $5,6 \%$ & $16,7 \%$ & $22,2 \%$ \\
\hline & \multirow{3}{*}{ Pseudoptosis } & Count & 5 & 4 & 9 \\
\hline & & $\%$ within Grade of ptosis & $55,6 \%$ & $44,4 \%$ & $100,0 \%$ \\
\hline & & $\%$ of Total & $13,9 \%$ & $11,1 \%$ & $25,0 \%$ \\
\hline & \multirow{3}{*}{ Total } & Count & 18 & 18 & 36 \\
\hline & & $\%$ within Grade of ptosis & $50,0 \%$ & $50,0 \%$ & $100,0 \%$ \\
\hline & & $\%$ of Total & $50,0 \%$ & $50,0 \%$ & $100,0 \%$ \\
\hline
\end{tabular}

Table 8: The statistical relationship between the grade of ptosis and postoperative complications (major and minor) is insignificant $(\mathrm{p}>0,05)$.

\begin{tabular}{|c|c|c|c|c|c|}
\hline \multicolumn{7}{|c|}{ Group Statistics } \\
\hline & Revision performed for & N & Mean & Std. Deviation & Std. Error Mean \\
\hline \multirow{2}{*}{$\begin{array}{c}\text { Implant } \\
\text { cc R }\end{array}$} & No & 31 & 211,29 & 61,182 & 10,989 \\
\cline { 2 - 6 } & Yes & 5 & 215,00 & 108,397 & 48,477 \\
\hline \multirow{2}{*}{$\begin{array}{c}\text { Implant } \\
\text { cc L }\end{array}$} & No & 31 & 212,90 & 59,816 & 10,743 \\
\cline { 2 - 6 } & Yes & 5 & 215,00 & 108,397 & 48,477 \\
\hline
\end{tabular}

Table 9: The statistical relationship between the implant volume and revision operations is insignificant $(\mathrm{p}>0,05)$.

\begin{tabular}{|c|c|c|c|c|c|}
\hline \multicolumn{7}{|c|}{ Group Statistics } \\
\hline \multirow{2}{*}{} & $\begin{array}{c}\text { Major postoperative } \\
\text { complications }\end{array}$ & $\mathrm{N}$ & Mean & Std. Deviation & Std. Error Mean \\
\hline \multirow{2}{*}{$\begin{array}{c}\text { Implant } \\
\text { cc R }\end{array}$} & No & 29 & 203,45 & 65,712 & 12,202 \\
\cline { 2 - 6 } & Yes & 7 & 246,43 & 68,357 & 25,836 \\
\hline $\begin{array}{c}\text { Implant } \\
\text { cc L }\end{array}$ & No & 29 & 205,17 & 64,566 & 11,990 \\
\cline { 2 - 6 } & Yes & 7 & 246,43 & 68,357 & 25,836 \\
\hline
\end{tabular}

Table 10: The statistical relationship between the implant volume and major postoperative complications is insignificant $(\mathrm{p}>0,05)$.

\begin{tabular}{|c|c|c|c|c|c|}
\hline \multicolumn{7}{|c|}{ Group Statistics } \\
\hline \multirow{2}{*}{} & $\begin{array}{c}\text { Minor postoperative } \\
\text { complications }\end{array}$ & $\mathrm{N}$ & Mean & Std. Deviation & Std. Error Mean \\
\hline \multirow{2}{*}{$\begin{array}{c}\text { Implant } \\
\text { cc R }\end{array}$} & No & 25 & 223,00 & 70,312 & 14,062 \\
\cline { 2 - 6 } & Yes & 11 & 186,36 & 55,186 & 16,639 \\
\hline $\begin{array}{c}\text { Implant } \\
\text { cc L }\end{array}$ & No & 25 & 225,00 & 68,465 & 13,693 \\
\cline { 2 - 6 } & Yes & 11 & 186,36 & 55,186 & 16,639 \\
\hline
\end{tabular}

Table 11: The statistical relationship between the implant volume and minor postoperative complications is insignificant $(\mathrm{p}>0,05)$.

\begin{tabular}{|c|c|c|c|c|c|}
\hline \multicolumn{7}{|c|}{ Group Statistics } \\
\hline \multirow{2}{*}{} & $\begin{array}{c}\text { Total postoperative } \\
\text { complications }\end{array}$ & $\mathrm{N}$ & Mean & Std. Deviation & Std. Error Mean \\
\hline $\begin{array}{c}\text { Implant } \\
\text { cc R }\end{array}$ & No & 18 & 213,89 & 70,826 & 16,694 \\
\cline { 2 - 6 } & Yes & 18 & 209,72 & 65,943 & 15,543 \\
\hline $\begin{array}{c}\text { Implant } \\
\text { cc L }\end{array}$ & No & 18 & 216,67 & 68,599 & 16,169 \\
\cline { 2 - 6 } & Yes & 18 & 209,72 & 65,943 & 15,543 \\
\hline
\end{tabular}

Table 12: The statistical relationship between the implant volume and total postoperative complications (both major and minor) is insignificant ( $\mathrm{p}>0,05)$.

\begin{tabular}{|c|c|c|c|c|c|}
\hline \multicolumn{6}{|c|}{ Crosstab } \\
\hline & & & \multicolumn{2}{|c|}{$\begin{array}{l}\text { Revision performed } \\
\text { for }\end{array}$} & \multirow[t]{2}{*}{ Total } \\
\hline & & & No & Yes & \\
\hline \multirow{6}{*}{$\begin{array}{c}\text { Primary } \\
\text { (P) or } \\
\text { Secondary } \\
\text { (S) }\end{array}$} & \multirow{3}{*}{ Primary } & Count & 25 & 3 & 28 \\
\hline & & $\begin{array}{c}\% \text { within Primary }(\mathrm{P}) \\
\text { Secondary }(\mathrm{S})\end{array}$ & $89,3 \%$ & $10,7 \%$ & $100,0 \%$ \\
\hline & & $\%$ of Total & $69,4 \%$ & $8,3 \%$ & $77,8 \%$ \\
\hline & \multirow{3}{*}{ Secondary } & Count & 6 & 2 & 8 \\
\hline & & $\begin{array}{c}\% \text { within Primary }(\mathrm{P}) \\
\text { Secondary }(\mathrm{S})\end{array}$ & $75,0 \%$ & $25,0 \%$ & $100,0 \%$ \\
\hline & & $\%$ of Total & $16,7 \%$ & $5,6 \%$ & $22,2 \%$ \\
\hline \multirow{3}{*}{\multicolumn{2}{|c|}{ Total }} & Count & 31 & 5 & 36 \\
\hline & & $\begin{array}{c}\% \text { within Primary }(\mathrm{P}) \\
\text { Secondary }(\mathrm{S})\end{array}$ & $86,1 \%$ & $13,9 \%$ & $100,0 \%$ \\
\hline & & $\%$ of Total & $86,1 \%$ & $13,9 \%$ & $100,0 \%$ \\
\hline
\end{tabular}

Table 13: The statistical relationship between groups (primary or secondary) and revision operations is insignificant $(\mathrm{p}>0,05)$. 


\begin{tabular}{|c|c|c|c|c|c|}
\hline \multicolumn{6}{|c|}{ Crosstab } \\
\hline & & & \multicolumn{2}{|c|}{$\begin{array}{l}\text { Major postoperative } \\
\text { complications }\end{array}$} & \multirow{2}{*}{ Total } \\
\hline & & & No & Yes & \\
\hline \multirow{6}{*}{$\begin{array}{l}\text { Primary } \\
\text { (P) or } \\
\text { Secondary } \\
\text { (S) }\end{array}$} & \multirow{3}{*}{ Primary } & Count & 25 & 3 & 28 \\
\hline & & $\begin{array}{c}\% \text { within Primary }(\mathrm{P}) \\
\text { Secondary }(\mathrm{S})\end{array}$ & $89,3 \%$ & $10,7 \%$ & $100,0 \%$ \\
\hline & & $\%$ of Total & $69,4 \%$ & $8,3 \%$ & $77,8 \%$ \\
\hline & \multirow{3}{*}{ Secondary } & Count & 4 & 4 & 8 \\
\hline & & $\begin{array}{c}\% \text { within Primary }(\mathrm{P}) \\
\text { Secondary }(\mathrm{S})\end{array}$ & $50,0 \%$ & $50,0 \%$ & $100,0 \%$ \\
\hline & & $\%$ of Total & $11,1 \%$ & $11,1 \%$ & $22,2 \%$ \\
\hline \multirow{3}{*}{\multicolumn{2}{|c|}{ Total }} & Count & 29 & 7 & 36 \\
\hline & & $\begin{array}{c}\% \text { within Primary }(\mathrm{P}) \\
\text { Secondary }(\mathrm{S})\end{array}$ & $80,6 \%$ & $19,4 \%$ & $100,0 \%$ \\
\hline & & $\%$ of Total & $80,6 \%$ & $19,4 \%$ & $100,0 \%$ \\
\hline
\end{tabular}

Table 14: The statistical relationship between groups (primaryor secondary) and major postoperative complications is significant $(\mathrm{p}<0,05)$.

\begin{tabular}{|c|c|c|c|c|c|}
\hline \multicolumn{6}{|c|}{ Crosstab } \\
\hline & & & \multicolumn{2}{|c|}{$\begin{array}{l}\text { Minor postoperative } \\
\text { complications }\end{array}$} & \multirow[t]{2}{*}{ Total } \\
\hline & & & No & Yes & \\
\hline \multirow{6}{*}{$\begin{array}{l}\text { Primary } \\
\text { (P) or } \\
\text { Secondary } \\
\text { (S) }\end{array}$} & \multirow{3}{*}{ Primary } & Count & 17 & 11 & 28 \\
\hline & & $\begin{array}{c}\% \text { within Primary }(\mathrm{P}) \\
\text { Secondary }(\mathrm{S})\end{array}$ & $60,7 \%$ & $39,3 \%$ & $100,0 \%$ \\
\hline & & $\%$ of Total & $47,2 \%$ & $30,6 \%$ & $77,8 \%$ \\
\hline & \multirow{3}{*}{ Secondary } & Count & 8 & 0 & 8 \\
\hline & & $\begin{array}{c}\% \text { within Primary }(\mathrm{P}) \\
\text { Secondary }(\mathrm{S})\end{array}$ & $100,0 \%$ & $0,0 \%$ & $100,0 \%$ \\
\hline & & $\%$ of Total & $22,2 \%$ & $0,0 \%$ & $22,2 \%$ \\
\hline \multirow{3}{*}{\multicolumn{2}{|c|}{ Total }} & Count & 25 & 11 & 36 \\
\hline & & $\begin{array}{c}\% \text { within Primary }(\mathrm{P}) \\
\text { Secondary }(\mathrm{S})\end{array}$ & $69,4 \%$ & $30,6 \%$ & $100,0 \%$ \\
\hline & & $\%$ of Total & $69,4 \%$ & $30,6 \%$ & $100,0 \%$ \\
\hline
\end{tabular}

Table 15: The statistical relationship between groups (primary or secondary) and minor postoperative complications is insignificant $(\mathrm{p}>0,05)$.

\begin{tabular}{|c|c|c|c|c|c|}
\hline \multicolumn{6}{|c|}{ Crosstab } \\
\hline & & & \multicolumn{2}{|c|}{$\begin{array}{l}\text { Minor postoperative } \\
\text { complications }\end{array}$} & \multirow[t]{2}{*}{ Total } \\
\hline & & & No & Yes & \\
\hline \multirow{6}{*}{$\begin{array}{l}\text { Primary } \\
\text { (P) or } \\
\text { Secondary } \\
\text { (S) }\end{array}$} & \multirow{3}{*}{ Primary } & Count & 14 & 14 & 28 \\
\hline & & $\begin{array}{c}\% \text { within Primary }(\mathrm{P}) \\
\text { Secondary }(\mathrm{S})\end{array}$ & $50,0 \%$ & $50,0 \%$ & $100,0 \%$ \\
\hline & & $\%$ of Total & $38,9 \%$ & $38,9 \%$ & $77,8 \%$ \\
\hline & \multirow{3}{*}{ Secondary } & Count & 4 & 4 & 8 \\
\hline & & $\begin{array}{c}\% \text { within Primary }(\mathrm{P}) \\
\text { Secondary }(\mathrm{S})\end{array}$ & $50,0 \%$ & $50,0 \%$ & $100,0 \%$ \\
\hline & & $\%$ of Total & $11,1 \%$ & $11,1 \%$ & $22,2 \%$ \\
\hline \multirow{3}{*}{\multicolumn{2}{|c|}{ Total }} & Count & 18 & 18 & 36 \\
\hline & & $\begin{array}{c}\% \text { within Primary }(\mathrm{P}) \\
\text { Secondary }(\mathrm{S})\end{array}$ & $50,0 \%$ & $50,0 \%$ & $100,0 \%$ \\
\hline & & $\%$ of Total & $50,0 \%$ & $50,0 \%$ & $100,0 \%$ \\
\hline
\end{tabular}

Table 16: The statistical relationship between groups (primary or secondary) and total postoperative complications (both major and minor) is insignificant $(\mathrm{p}>0,05)$.

\section{Discussion}

Surgeons prefer to insert breast implants under the muscle in many of their augmentation cases. This option is also valid when additional mastopexy is considered. Thus, bi-planar mastopexy-augmentation may be indicated in selected cases. As an example, we have observed that the maneuver to fix the breast tissue to the pectoral fascia during vertical mammaplasty, not only increases the tightness of the breast tissue and the tension on the suture line, but also restricts the volume of the submammarial pocket for the implant. As a result, the implant is pushed downward and outward, making the suture line tighter, especially on the lower breast pole; this observation is especially important in the context of larger implants. Consequently, in selected cases we inserted the implants into the submuscular plane after fixing the glandular tissue to a higher level on the subglandular plane (hence bi-planar mastopexy-augmentation). The increased tension on the suture line due to the Lejour fixing suture and implantation should be taken into consideration when planning the preoperative markings; especially in cases involving firmer breasts. Other example is the formation of capsular contracture in the subglandular plane after the primary operation and desire to avoid the same plane.

In two-plane mastopexy-augmentation, positioning the implant on a desired higher level may enable the surgeon to cover the implant with muscle superiorly and superomedian dermoglandular or dermofat flap inferiorly, decreasing the risk of implant exposure during wound healing. We also believe that the submuscular plane enables the surgeon to use smooth surface implants, which can slide inside and adapt to postoperative changes in the breast shape.

Beale et al., [11] investigated Rohrich's 83 mastopexy-augmentation cases retrospectively and determined a number of important safety criteria, these included precise preoperative marking, the use of $8 \mathrm{~cm}$ vertical limbs with broad pedicle base, limited undermining of thick skin flaps, small subpectoral implants $(<200 \mathrm{ml})$, and the movement of nipple no more than $4 \mathrm{~cm}$. They stated that the use of smaller subpectoral implants results in minimal skin flap and nipple loss. They also advocated minimal capsule removal in secondary cases so as not to compromise the vascular network of the flaps. We agree with these points and additionally, we would like to contribute that the capsule is an ideal tissue to fix the lifted breast to; this results in a safer wound closure, especially in cases that involve closing the skin just above the implant surface. In cases where there is severe capsule contraction in the subglandular plane, a second plane (the submuscular plane) would be an ideal place to insert the implant. Furthermore, the capsule itself would be a very effective tissue on which to hold the lifting sutures. Therefore, the two-plane method may be a suitable option to manage secondary cases with severe capsule contractions.

In our study, the rates of revision operations and postoperative major complications were $13.89 \%$ and $19.44 \%$, respectively. Khavanin et al., [3] performed a meta-analysis of single stage mastopexy-augmentation by reviewing 23 studies. They found the approximate reoperation rate to be $10.7 \%(6.7 \%-15.4 \%)$ and the complication rate to be $13.1 \%(6.7 \%-21.3 \%)$. Our results are comparable with these findings. However, we believe that the patients should be informed profoundly about all potential problems after one stage mastopexy-augmentation operations. 
We observed that asymmetries and bad scar formation were the most frequently reported complications. Most asymmetries were due to tissue laxity and sagging, especially on the inferior pole of the breast. This has the potential to create a dilemma in that the tightness of the lateral pillars may result in wound healing issues, even as severe as implant exposure; on the other hand, the laxity of the lateral pillars may cause sagging. Excision of the sagged area as a secondary procedure is usually sufficient to obtain the desired shape.

We found that major complications were significantly more frequent in the secondary group; this could be due to the larger implant volumes in the secondary group. However, there was no statistical correlation between the implant volumes and the major complications. Nevertheless, it should be taken into consideration that the main limitation of this study was the lower number of cases in the secondary group. On the other hand, Beale et al., [11] recommended smaller implants to avoid the aforementioned complications. In spite of small study population of the second group, we recommend caution for the secondary mastopexy-augmentation cases.

\section{Conclusion}

We believe that bi-planar mastopexy-augmentation, and coverage of the implants with median dermofat or dermoglandular tissue, could be performed in selected cases. However, potential postoperative problems should be taken into considerations and more caution is needed for secondary cases.

\section{Conflicts of Interest and Source of Funding:}

The authors declare that they have no conflict of interest to disclose.

\section{References}

1. Spear SL (2006) Augmentation/Mastopexy: "surgeon, beware". Plast Reconstr Surg 118: 133-134.

2. Spear SL, Boehmler JH 4th, Clemens MW (2006) Augmentation/mastopexy: A 3-year review of a single surgeon's practice. Plast Reconstr Surg 118:136-147.

3. Khavanin N, Jordan SW, Rambachan A, Kim JY (2014) A systematic review of single-stage augmentation-mastopexy. Plast Reconstr Surg 134: 922-931.

4. Castello MF, Silvestri A, Nicoli F, Dashti T, Han S, et al. (2014) Augmentation mammoplasty/mastopexy: Lessons learned from 107 aesthetic cases. Aesthetic Plast Surg 38: 896-907.

5. Stevens WG, Stoker DA, Freeman ME, Quardt SM, Hirsch EM, et al. (2006) Is one-stage breast augmentation with mastopexy safe and effective? A review of 186 primary cases. Aesthet Surg J 26: 674-681.

6. Swanson E (2013) Prospective comparative clinical evaluation of 784 consecutive cases of breast augmentation and vertical mammaplasty, performed individually and in combination. Plast Reconstr Surg 132: 30-45.

7. Lee MR, Unger JG, Adams WP Jr. (2014) The tissue-based triad: A process approach to augmentation mastopexy. Plast Reconstr Surg 134: 215-225.

8. Stevens WG, Macias LH, Spring M, Stoker DA, Chacón CO, et al. (2014) One-Stage Augmentation Mastopexy: A Review of 1192 Simultaneous Breast Augmentation and Mastopexy Procedures in 615 Consecutive Patients. Aesth Surg J 34: 723-732.

9. Lejour M (1994) Vertical mammaplasty and liposuction of the breast. Plast Reconstr Surg 94: 100-114.

10. Lejour M (1999) Vertical mammaplasty: Update and appraisal of late results. Plast Reconstr Surg 104: 771-781.

11. Beale EW, Ramanadham S, Harrison B, Rasko Y, Armijo B, et al. (2014) Achieving predictability in augmentation mastopexy. Plast Reconstr Surg 133: 284-292. 


\section{Hit \\ ндтар}

Journal of Anesthesia \& Clinical Care

Journal of Addiction \& Addictive Disorders

Advances in Microbiology Research

Advances in Industrial Biotechnology

Journal of Agronomy \& Agricultural Science

Journal of AIDS Clinical Research \& STDs

Journal of Alcoholism, Drug Abuse \& Substance Dependence

Journal of Allergy Disorders \& Therapy

Journal of Alternative, Complementary \& Integrative Medicine

Journal of Alzheimer's \& Neurodegenerative Diseases

Journal of Angiology \& Vascular Surgery

Journal of Animal Research \& Veterinary Science

Archives of Zoological Studies

Archives of Urology

Journal of Atmospheric \& Earth-Sciences

Journal of Aquaculture \& Fisheries

Journal of Biotech Research \& Biochemistry

Journal of Brain \& Neuroscience Research

Journal of Cancer Biology \& Treatment

Journal of Cardiology: Study \& Research

Journal of Cell Biology \& Cell Metabolism

Journal of Clinical Dermatology \& Therapy

Journal of Clinical Immunology \& Immunotherapy

Journal of Clinical Studies \& Medical Case Reports

Journal of Community Medicine \& Public Health Care

Current Trends: Medical \& Biological Engineering

Journal of Cytology \& Tissue Biology

Journal of Dentistry: Oral Health \& Cosmesis

Journal of Diabetes \& Metabolic Disorders

Journal of Dairy Research \& Technology

Journal of Emergency Medicine Trauma \& Surgical Care

Journal of Environmental Science: Current Research

Journal of Food Science \& Nutrition

Journal of Forensic, Legal \& Investigative Sciences

Journal of Gastroenterology \& Hepatology Research

Journal of Gerontology \& Geriatric Medicine
Journal of Genetics \& Genomic Sciences

Journal of Hematology, Blood Transfusion \& Disorders

Journal of Human Endocrinology

Journal of Hospice \& Palliative Medical Care

Journal of Internal Medicine \& Primary Healthcare

Journal of Infectious \& Non Infectious Diseases

Journal of Light \& Laser: Current Trends

Journal of Modern Chemical Sciences

Journal of Medicine: Study \& Research

Journal of Nanotechnology: Nanomedicine \& Nanobiotechnology

Journal of Neonatology \& Clinical Pediatrics

Journal of Nephrology \& Renal Therapy

Journal of Non Invasive Vascular Investigation

Journal of Nuclear Medicine, Radiology \& Radiation Therapy

Journal of Obesity \& Weight Loss

Journal of Orthopedic Research \& Physiotherapy

Journal of Otolaryngology, Head \& Neck Surgery

Journal of Protein Research \& Bioinformatics

Journal of Pathology Clinical \& Medical Research

Journal of Pharmacology, Pharmaceutics \& Pharmacovigilance

Journal of Physical Medicine, Rehabilitation \& Disabilities

Journal of Plant Science: Current Research

Journal of Psychiatry, Depression \& Anxiety

Journal of Pulmonary Medicine \& Respiratory Research

Journal of Practical \& Professional Nursing

Journal of Reproductive Medicine, Gynaecology \& Obstetrics

Journal of Stem Cells Research, Development \& Therapy

Journal of Surgery: Current Trends \& Innovations

Journal of Toxicology: Current Research

Journal of Translational Science and Research

Trends in Anatomy \& Physiology

Journal of Vaccines Research \& Vaccination

Journal of Virology \& Antivirals

Archives of Surgery and Surgical Education

Sports Medicine and Injury Care Journal

International Journal of Case Reports and Therapeutic Studies

Journal of Ecology Research and Conservation Biology

Submit Your Manuscript: http://www.heraldopenaccess.us/Online-Submission.php 